\title{
Temperature dependent photoluminescence from porous silicon nanostructures: Quantum confinement and oxide related transitions
}

\author{
Mallar Ray, ${ }^{1, a)}$ Nil Ratan Bandyopadhyay, ${ }^{1}$ Ujjwal Ghanta, ${ }^{1}$ Robert F. Klie, ${ }^{2}$ \\ Ashit Kumar Pramanick, ${ }^{3}$ Samaresh Das, ${ }^{4}$ Samit K. Ray, ${ }^{4}$ and Syed Minhaz Hossain ${ }^{5}$ \\ ${ }^{1}$ School of Materials Science and Engineering, Bengal Engineering and Science University, Shibpur, \\ Howrah 711103, West Bengal, India \\ ${ }^{2}$ Department of Physics, University of Illinois at Chicago, Chicago 60607, Illinois, USA \\ ${ }^{3}$ Materials Science and Technology Division, National Metallurgical Laboratory, Jamshedpur 831007, \\ Jharkhand, India \\ ${ }^{4}$ Department of Physics and Meteorology, Indian Institute of Technology, Kharagpur: 721 302, \\ West Bengal, India \\ ${ }^{5}$ Department of Physics, Bengal Engineering and Science University, Shibpur, Howrah 711103 , \\ West Bengal, India
}

(Received 7 July 2011; accepted 21 September 2011; published online 4 November 2011)

\begin{abstract}
Temperature dependent photoluminescence (PL) spectroscopy along with structural investigations of luminescent porous Si enable us to experimentally distinguish between the relative contributions of band-to-band and oxide interface mediated electronic transitions responsible for light emission from these nanostructures. Porous Si samples formed using high current densities $\left(J \geq 80 \mathrm{~mA} / \mathrm{cm}^{2}\right)$ have large porosities $(P \geq 85 \%)$ and consequently smaller $(\sim 1-6 \mathrm{~nm})$ average crystallite sizes. The PL spectra of these high porosity samples are characterized by multiple peaks. Two dominant peaks — one in the blue regime and one in the yellow/orange regime, along with a very low intensity red/NIR peak, are observed for these samples. The high energy peak position is nearly independent of temperature, whereas the yellow/orange peak red-shifts with increasing temperature. Both the peaks blue shift with ageing and with increasing porosity. The intensity of the blue peak increases whereas the yellow/orange peak decreases with increasing temperature, while the intensity and peak position of the very low intensity red/NIR peak appears to be unaffected by temperature, porosity, and ageing. The low porosity samples $(P \leq 60 \%)$ on the other hand exhibit a single PL peak whose intensity decreases and exhibits a very small red spectral shift with increase in temperature. From the variation of intensity and PL peak positions, it is established that both quantum confinement of excitons and oxide related interfacial defect states play dominant role in light emission from porous $\mathrm{Si}$ and it is possible to qualitatively distinguish and assign their individual contributions.
\end{abstract} (C) 2011 American Institute of Physics. [doi:10.1063/1.3657771]

\section{INTRODUCTION}

Light emission form porous Si has been one of the most well researched areas since the discovery of visible photoluminescence (PL) in 1990. ${ }^{1}$ Despite two decades of extensive research on various aspects of this promising material, the mechanism of light emission has hitherto remained controversial. $^{2-12}$ Though it is now more-or-less accepted that both quantum confinement of excitons, as well as localized defect states at the surface/interface of nanocrystalline $\mathrm{Si}$ (nc-Si) and the surrounding oxide layer have combined contributions towards visible PL from porous $\mathrm{Si}$, it has remained difficult to isolate the relative contributions of interfacial defects and size. ${ }^{7,10,12-15}$

In an attempt to understand the mechanism of luminescence from porous Si structures, temperature dependent PL studies have been performed by several groups but completely contradictory results are reported in literature. Rongchuan et al. ${ }^{16}$ investigated the dependence of PL in porous Si over a temperature range of $85-457 \mathrm{~K}$ and reported that the wave-

${ }^{a)}$ Electronic mail: mray@matsc.becs.ac.in. length of the luminescent peak blue shifts but the PL band width remains unaltered with increase in temperature over this range. On the other hand, Ookubo and Sawada ${ }^{17}$ studied the red-band PL of porous Si for temperatures varying from 15 to $293 \mathrm{~K}$ and found that the PL peak red shifts and the width narrows, while the intensity increases until $\sim 70 \mathrm{~K}$ and then decreases slightly. Red shift of PL maxima and decrease of intensity after $100 \mathrm{~K}$ has also been observed by Finkbeiner and Weber. ${ }^{18}$ Gardelis et al. ${ }^{19}$ observed that PL peak consistently shifts to higher energies for temperature increasing from 3 to $20 \mathrm{~K}$, while Zeng et al. $^{20}$ reported that PL peak-energy shift with temperature has no fixed trend and varies from sample to sample, and even from sampling point to sampling point for the same layer. $\mathrm{Xu}$ et al. $^{21}$ observed that shift in PL peak energy with temperature is dependent on porosity. A recent study by $\mathrm{Xu}$ and Adachia ${ }^{22}$ demonstrated that low temperature PL spectra of porous $\mathrm{Si}$ are characterized by multiple-peak structure, which is explained by a configurational-coordinate model wherein the origin of multiple-peaks have been stated to be bulk-related, whereas the strength is associated to the surface oxide. Thus, a comprehensive and conclusive understanding of temperature dependence of PL from porous $\mathrm{Si}$ 
nanostructures and consequently the mechanism of luminescence is still not clear.

In this work, we show explicitly that primarily two competitive processes involving band-to-band and band-to-interface transitions are responsible for radiative emission from porous $\mathrm{Si}$ which account for the appearance of multiple peaks in high porosity (samples with smaller average crystallite size) porous $\mathrm{Si}$, whereas low porosity samples are characterized by a single peak. Low temperature PL spectra of these samples, particularly the ones having high porosity allow us to clearly distinguish between the contributions of the two competing processes.

\section{EXPERIMENTAL}

Porous Si samples were formed on $2-5 \Omega$-cm resistivity, p-type (100) orientation crystalline (c-) Si-wafers by anodization in $24 \%(\mathrm{w} / \mathrm{v}) \mathrm{HF}_{-} \mathrm{CH}_{3} \mathrm{OH}$ solution in teflon bath with special electrode geometry to form porous $\mathrm{Si}$ layers having uniform macromorphology. ${ }^{23}$ Varying formation-current density $(J)$ and time produced porous Si structures with different porosity and consequently different crystallite sizes. Porosity, defined as the fraction of void within the porous layer, was determined using the standard gravimetric technique ${ }^{3,24}$ and confirmed by the artificial neural network based model reported elsewhere. ${ }^{25}$ Although samples with different porosities were prepared and investigated, for the purpose of the present study, we concentrate on two sets of samples, one with porosity $\sim 60 \%$ (low-porosity samples) and the other set with porosities $>85 \%$ (high porosity samples).

Samples for high resolution transmission electron microscopy (HRTEM) were prepared by exfoliation of the porous layer by passing a high current pulse $\left(J=2 \mathrm{~A} / \mathrm{cm}^{2}\right)$ for a very short time $(\sim 1 \mathrm{~s})$ that caused the porous layer to detach from the Si substrate due to the enhanced strain generated. ${ }^{26}$ The loosely attached layer was then removed by an appropriately cut scotch tape and the crystallites were suspended in isopropanol. The samples were then drop casted on a carbon coated copper grid after thorough sonication and the images were recorded by a JEOL JEM 3010 operating at $300 \mathrm{kV}$. Atomic force microscopy (AFM) and scanning tunneling microscopy (STM) was performed using SPA 400, Seiko Japan. The structures of the exposed porous Si layers were investigated at room temperature, in a constant current-mode $(0.341 \mathrm{nA})$ with a tip diameter of $50 \mathrm{~nm}$. The porous structures were visualized by surface roughness across the scan area. The sensitivity of the tunnel current allowed detection of pores having diameters ranging from 0.5 to $1 \mathrm{~nm}$. AFM imaging (non-contact mode) of the colloids formed by exfoliation of the porous layers was carried out by depositing the colloid over freshly cleaved mica surface. Temperature dependent PL spectra were recorded by Triax 310 monochromator and a multichannel photomultiplier detector under UV excitation with $325 \mathrm{~nm}$ line of a $45 \mathrm{~mW} \mathrm{HeCd}$ laser. A thermoelectrically cooled cryostat allowed recording PL at temperatures as low as $10 \mathrm{~K}$. To ensure consistency, PL spectra at room temperature of all the samples were also recorded using a Horiba Jobin Yvon, Nanolog spectrofluorometer fitted with a xenon source.

\section{RESULTS AND DISCUSSIONS}

Figures 1(a)-1(d) are some of the representative HRTEM images of the exfoliated porous Si layers. The colloidal suspensions of exfoliated layer of $\mathrm{Si}$ nanocrystals (ncs) upon evaporation on a TEM grid forms extended agglomerates having an appearance of crystalline islands in amorphous background. Fig. 1(a) is the bright field image capturing a single crystal of a low porosity sample formed with $J=10 \mathrm{~mA} / \mathrm{cm}^{2}$ and etched for $5 \mathrm{~min}$ having porosity $\sim 60 \%$. As expected, the size of the crystallite captured here is large $(>7 \mathrm{~nm})$. Since a high current pulse $\left(\sim 2 \mathrm{~A} / \mathrm{cm}^{2}\right)$ is passed after the formation of porous layer to detach the porous film, a portion of the substrate just below the film also gets detached and that is reflected by the presence of chunk of c-Si as marked in Fig. 1(a).

The images shown in Figs. 1(b) and 1(c) are for the samples having porosities $\sim 88 \%$ and $91 \%$, respectively. Nonspherical crystallites with one of its dimensions $\sim 2 \mathrm{~nm}$ are clearly detectable. While Fig. 1(b) captures only one Si nc with aspect ratio $\sim 1.6$, randomly distributed Si ncs revealing the Si (111), (220), and the (311) lattice fringes are all detectable from Fig. 1(c). The selected area electron diffraction pattern (SADP) shown in Fig. 1(d) corresponds to the sample shown in Fig. 1(c). The SADP clearly shows rings of Si and the corresponding planes are (111), (220), and (311). The (331) plane is also visible (not marked). The nearly continuous rings are suggestive of random orientation of the neighboring nanocrystallites.

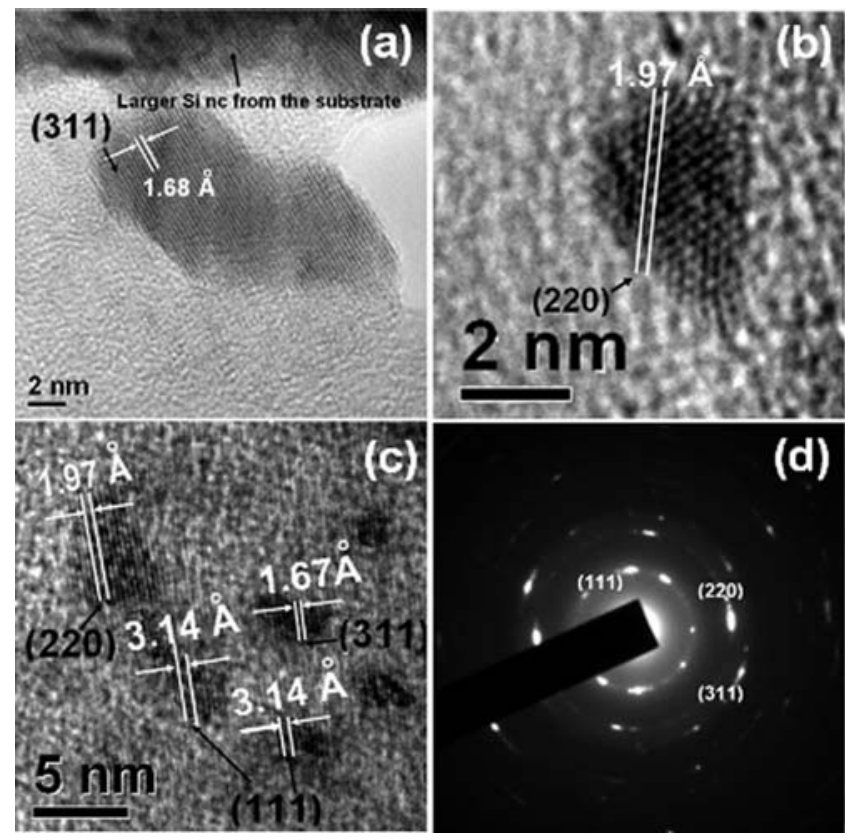

FIG. 1. HRTEM images of porous Si layers obtained by depositing the exfoliated layers on carbon coated copper grids. (a) Bright field image of a low porosity $(\sim 60 \%)$ sample formed with $J=10 \mathrm{~mA} / \mathrm{cm}^{2}$, etching time $=5 \mathrm{~min}$, and $[\mathrm{HF}]=24 \%$. A comparatively large crystallite along with a chunk detached from the substrate is also detected. (b) A non-spherical ncSi obtained from a sample prepared with $J=80 \mathrm{~mA} / \mathrm{cm}^{2}, t=15 \mathrm{~min}$, and $[\mathrm{HF}]=24 \%$. (c) Randomly oriented nc-Si with the (111), (220), and (311) exposed planes with even smaller dimensions obtained from sample prepared with $J=100 \mathrm{~mA} / \mathrm{cm}^{2}, t=15 \mathrm{~min}$, and $[\mathrm{HF}]=24 \%$. (d) SAD pattern of the sample shown in (c) reveals the same planes. 


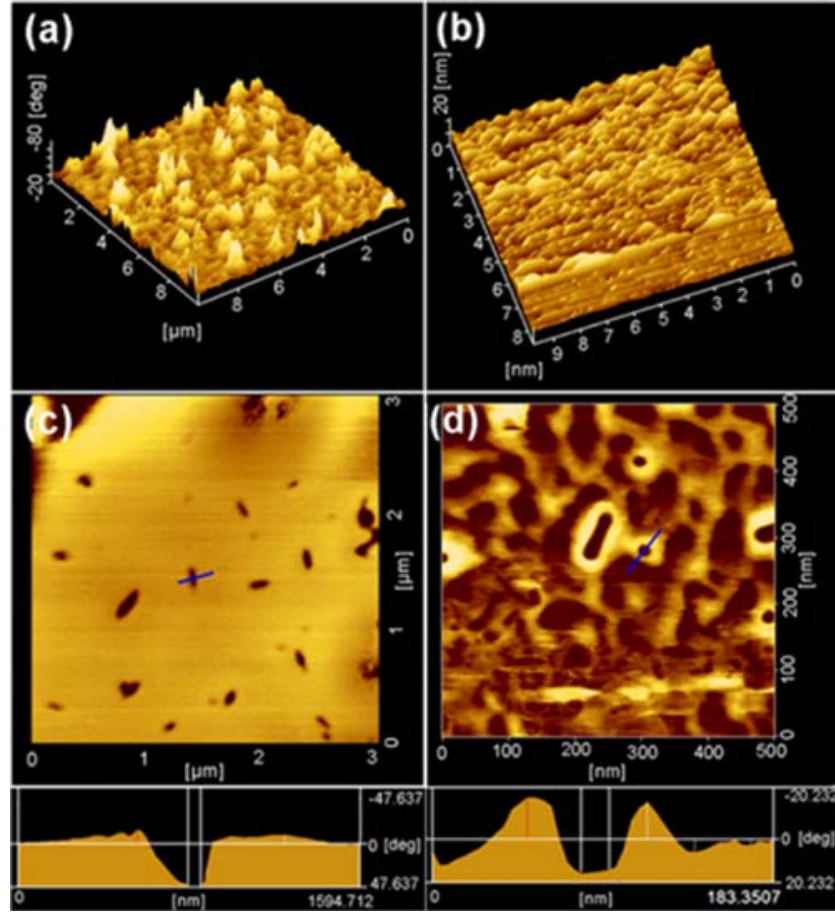

FIG. 2. (Color online) STM and AFM images of porous Si nanostructures. (a) and (b) are the STM images of as-grown, low $(\sim 60 \%)$ and high $(\sim 91 \%)$ porosity porous Si samples, respectively; (c) depicts the topographic image and the line profile of the marked particle, for the ncs obtained from the high porosity samples by exfoliation followed by deposition on cleaved mica; and (d) phase contrast AFM image of the sample shown in (c) depicting the formation of thick oxide shell, which manifest as humps in the line profile shown below the image.

Typical STM images of as-grown porous Si layers on substrates with porosities $\sim 60 \%$ and $91 \%$ are shown in Figs. 2(a) and 2(b), respectively. It is clear from these images that, although both the samples are characterized by "hills and valleys" type of structure, the sizes of the crystallites and the surface morphology are strongly dependent on the formation parameters and consequently on the porosities. ${ }^{5-7}$ The samples formed with larger current density $\left(J=100 \mathrm{~mA} / \mathrm{cm}^{2}\right)$ have much smaller nc-Si compared to the one formed with lower value of $J\left(10 \mathrm{~mA} / \mathrm{cm}^{2}\right)$.

In an attempt to account for the general structural features and sizes of the nc-Si and to distinguish between the $\mathrm{c}-\mathrm{Si}$ and the corresponding oxide phase, AFM imaging of the exfoliated layers deposited on mica were carried out. Figs. 2(c) and 2(d) show the topographic and the phase contrast AFM images of a high porosity sample (porosity 91\%) along with the line profiles of the particles marked in figures. The size segregation indicated in Figs. 2(c) and 2(d) was achieved through repeated sonication and centrifugation. Following the method we reported earlier, ${ }^{27}$ estimation of the core and shell dimensions of each particle identifiable in Figs. 2(c) and 2(d) was done from line profile analyses. Here, it may be noted that the dimension of the individual $\mathrm{Si}$ ncs are obtained much more precisely from the vertical topography data, ${ }^{28}$ and hence the sizes of the ncs were estimated from the $z$-height rather than the lateral spread. The cross-sections of the Si ncs in Fig. 2(c) were found to range between $\sim 1-6 \mathrm{~nm}$. It is clear from the phase image shown in
Fig. 2(d) that the sample consists of both nanorods and nanodots (dominated by non-spherical nanorod-like structures) of nc-Si surrounded by a thick oxide shell which is also reflected in the corresponding line profile. The region over which there is no variation of elastic/viscoelastic property represents c-Si core and the surrounding brighter region represents $\mathrm{Si}$ oxide with perceptible strain concentrations at the extended and diffused interface. ${ }^{27,29}$ Further, it is evident from the line profiles that the slopes on either side of the crystalline core are not identical, indicating that the amorphous shell is not evenly spread around the core. These structural observations are important and corroborates with our later findings, wherein, we see that the oxide shell as well as the nc-Si core have significant roles in determining the mechanism of radiative recombination in these nanostructures.

Before discussing the temperature dependent PL spectra of different porous Si samples, it is important to mention that a blue shift in the $S$-band PL peak with time was observed for all the samples (not shown here) after storing them in ambient air (or DI water) at room temperature for a period of one month. Similar observations have previously been reported and explained in terms of both surface passivation phenomena and carrier confinement. ${ }^{30-32}$ In addition, room temperature PL measurements of samples prepared with different formation parameters revealed that high porosity samples formed with higher current densities $\left(J \geq 80 \mathrm{~mA} / \mathrm{cm}^{2}\right)$ exhibit multiple peaks and/or other features in the PL spectra. To illustrate this point, the room temperature PL characteristics of the samples prepared with $J=80$ and $100 \mathrm{~mA} / \mathrm{cm}^{2},[\mathrm{HF}]=24 \%$, and etched for $15 \mathrm{~min}$ were recorded up to $925 \mathrm{~nm}$ and presented in Fig. 3.

The appearance of a distinct second peak at lower energy $(\sim 775-800 \mathrm{~nm})$ along with splitting of the high energy peaks (at $\sim 450$ and $490 \mathrm{~nm}$ for $J=100$ and at $\sim 425$, 468 , and $550 \mathrm{~nm}$ for $J=80 \mathrm{~mA} / \mathrm{cm}^{2}$, respectively) are evident from Fig. 3. The observation is indicative of different mechanisms involved in radiative transitions from porous $\mathrm{Si}$ nanostructures and propelled the temperature dependent

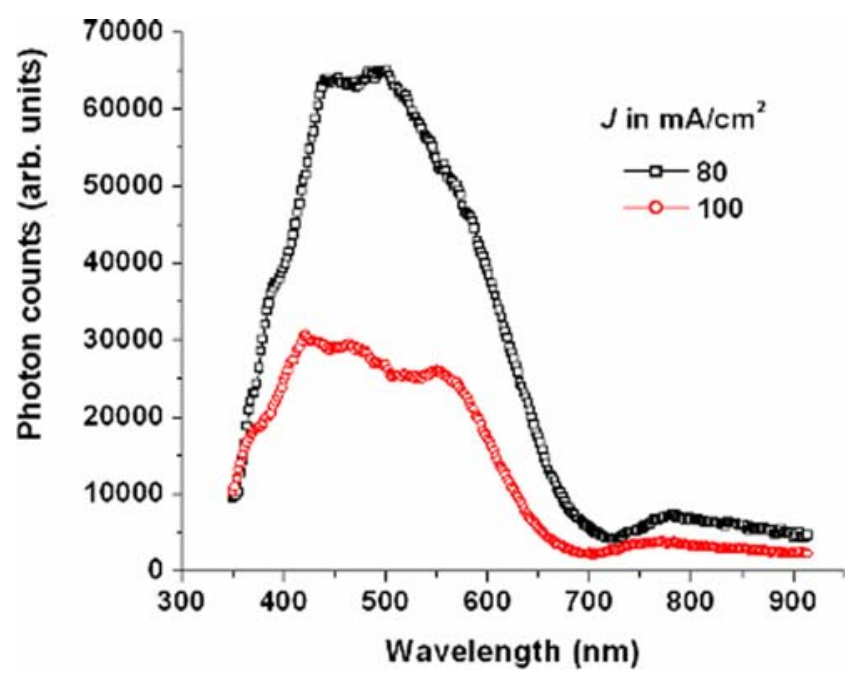

FIG. 3. (Color online) Room temperature PL spectra of high porosity samples prepared with $J=80$ and $100 \mathrm{~mA} / \mathrm{cm}^{2}, t=15 \mathrm{~min}$, and $[\mathrm{HF}]=24 \%$ showing the appearance of multiple peaks. 
study discussed below, in an attempt to gain an insight about the individual contributions of the different possible transition processes.

Figures 4(a)-4(d) show the variation of PL spectra of the samples prepared on $2-5 \Omega$-cm resistivity wafers with different formation parameters and consequently having different porosities, for temperature varying from $10 \mathrm{~K}$ to $300 \mathrm{~K}$. All the samples were analyzed after thorough rinsing in DI water, followed by storage in clean ambient atmosphere for different time intervals. It may be noted here that multiple peaks for these samples did not appear under asprepared condition. A weak PL signal at around $800 \mathrm{~nm}$ at room temperature was obtained.

It is clear from Fig. 4(a) that the position of the 1st peak (or more precisely, the high energy edge of the flattened peak at higher temperatures) is almost invariant under temperature change and remains nearly fixed at $\sim 462 \mathrm{~nm}$ $(2.68 \mathrm{eV})$, whereas the 2 nd peak exhibits a red spectral shift from $612 \mathrm{~nm}(2.03 \mathrm{eV})$ to $668 \mathrm{~nm}(1.86 \mathrm{eV})$ with increase in temperature from 10 to $300 \mathrm{~K}$. The intensity of the peaks on the other hand increases for the high energy peak and decreases for the low energy peak for the same variation in temperature. However, the position and intensity of the apparent $3 \mathrm{rd}$ peak at $800 \mathrm{~nm}$ remains constant. In addition, the width of the PL curve for the higher energy peak widens with increasing temperature and at room temperature the sharp peak starts flattening.

In an attempt to understand the role of ambient oxidation, the sample was allowed to age for 1 month in a covered Petri dish inside a clean fume hood and the temperature dependence of PL measurements were carried out. The spectra of this aged sample for the same variation in temperature are given in Fig. 4(b). Three distinct peaks can be clearly identified: (1) a high energy peak, henceforth referred as the 1st/blue energy peak, (2) a peak in the intermediate energy regime of the visible spectrum, referred as the 2nd/yellow-orange peak, and (3) a $3 \mathrm{rd} /$ red-NIR peak fixed at $\sim 800 \mathrm{~nm}(1.55 \mathrm{eV})$. The 1 st peak appears as a kink at a more-or-less constant energy $\sim 456 \mathrm{~nm}$ $(2.72 \mathrm{eV})$ and is distinctly discernable only at low temperatures (below $120 \mathrm{~K}$ ). Similar to the 1 day aged sample, here too the 2nd peak red shifts with increase in temperature but in this case at temperatures above $120 \mathrm{~K}$, the 1 st and the $2 \mathrm{nd}$ peaks tend to merge to form a plateau and become indistinguishable at around room temperature as shown in Fig. 4(b). In addition, comparing the PL spectra of the 1 day and 1 month aged samples [Figs. 4(a) and 4(b)], it is evident that both the 1 st and the 2 nd peaks exhibit blue shift with ageing but the third peak remains fixed at $\sim 800 \mathrm{~nm}$.

All samples prepared with $J \geq 80 \mathrm{~mA} / \mathrm{cm}^{2}$ exhibited multiple peaks and exactly similar trends in the variation of
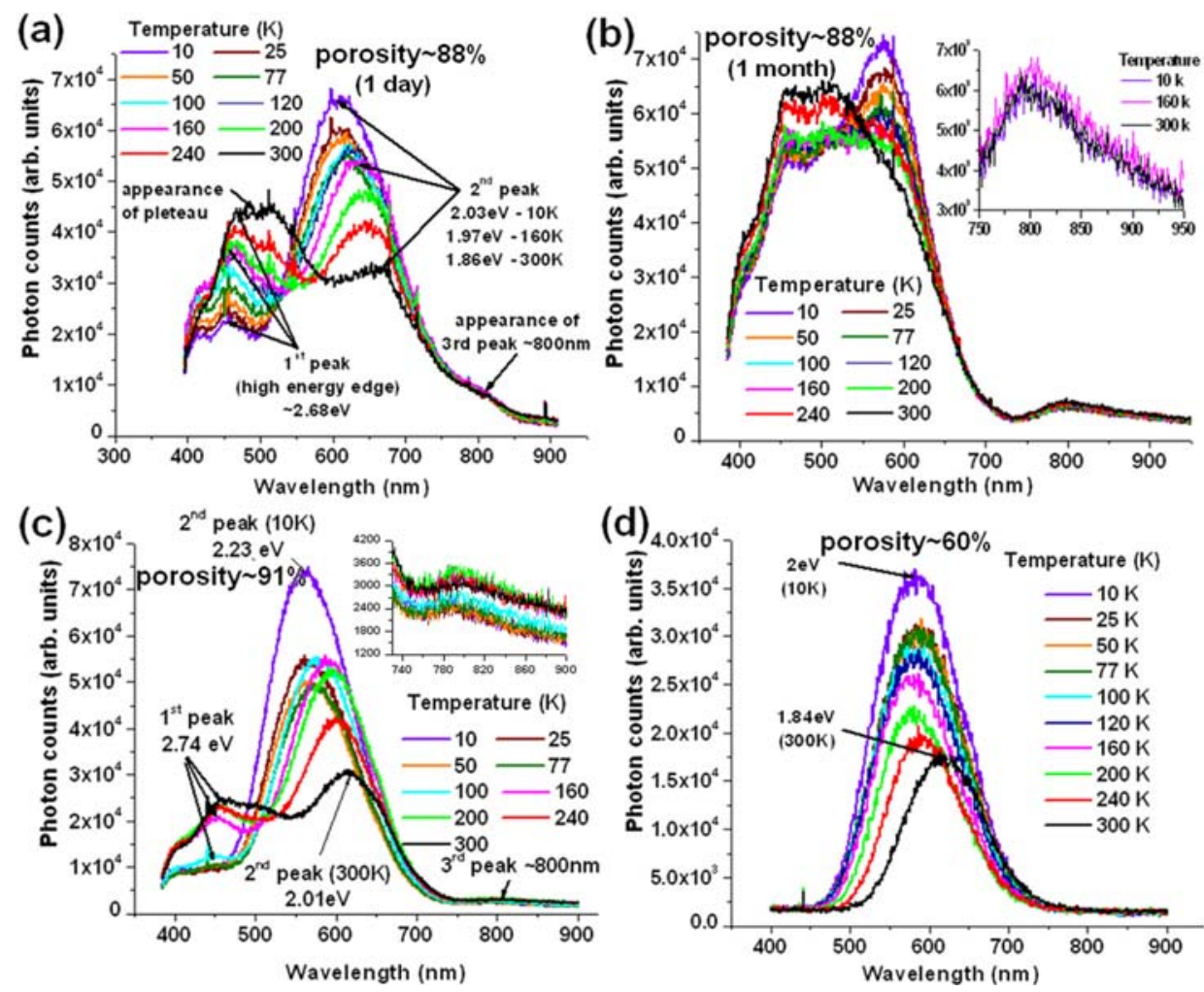

FIG. 4. (Color online) Temperature dependent PL characteristics of porous Si samples having different porosities for temperatures varying from 10-300 K. (a) Sample having porosity $88 \%$, prepared with $J=80 \mathrm{~mA} / \mathrm{cm}^{2}, t=15 \mathrm{~min}$, and $[\mathrm{HF}]=24 \%$ and dried in clean atmosphere for 1 day. Two distinct peaks, one in the blue region (indicated as 1st peak) and one in the yellow/orange region (depicted as 2nd peak) of the visible spectrum, are clearly visible. A third peak seems to appear at $\sim 800 \mathrm{~nm}$. (b) PL spectra of the same sample after 1 month ageing showing blue shift of the 1 st and the 2 nd peaks. The inset clearly shows the formation of a $3 \mathrm{rd}$ peak at $\sim 800 \mathrm{~nm}$. (c) Sample prepared with $J=100 \mathrm{~mA} / \mathrm{cm}^{2}, t=15 \mathrm{~min}$, [HF] $=24 \%$, and having porosity $\sim 91 \%$. PL spectra were recorded after 1 day of preparation. Similar trends as the samples shown in (a) and (b) are clear. However, since this is a higher porosity sample, the 1 st and the 2 nd peaks are further blue shifted. The inset is the magnified 3rd peak appearing in the red-NIR regime. (d) Low porosity sample having porosity $\sim 60 \%$ showing a red shift of the PL peak beyond $200 \mathrm{~K}$ with increase in temperature and absence of features (like peak splitting, etc. as observed in the high porosity samples) over the entire temperature range. 
peak position and intensity was observed. However, $J$ had to be kept $<120 \mathrm{~mA} / \mathrm{cm}^{2}$ (as the samples were electropolished for $J \geq 120 \mathrm{~mA} / \mathrm{cm}^{2}$, i.e., it exceeded the critical current density for pore formation). To re-affirm this point, the variation of PL characteristics with temperature of a sample prepared with $J=100 \mathrm{~mA} / \mathrm{cm}^{2}$, having porosity $\sim 91 \%$, is shown in Fig. 4(c).

As mentioned earlier, low temperature PL measurements were also carried out for other sets of samples prepared with different formation parameters but those prepared with lower $J$ values, and consequently having lower porosities did not show multiple peaks as shown in Fig. 4(d) for a representative sample having porosity $\sim 60 \%$, prepared with formation parameters: $J=10 \mathrm{~mA} / \mathrm{cm}^{2}, t=5 \mathrm{~min}$, and $[\mathrm{HF}]=24 \%$. It may be mentioned here that temperature dependent PL spectra of the 1 day aged sample with porosity $88 \%$ and the low porosity sample $(P=60 \%)$ were also recorded for a different power of the excitation energy by placing a glass slide (which reduced the incident power on the sample surface from $42 \mathrm{~mW}$ to 18 $\mathrm{mW}$ ) and exactly the same trend in variation of the spectra was obtained with reduced intensity.

From the findings of temperature dependent PL characteristics, the following general features can be summarized:

multiple peaks appear in the PL spectrum for high porosity samples $(P \geq 85 \%)$;

two dominant peaks are observed-one in the blue regime (1st peak) and one in the yellow/orange regime (2nd peak), along with a very low intensity 3rd peak in the red/NIR region;

(iii) the high energy peak position is almost independent of temperature for a given sample;

(iv) the low energy peak red-shifts with increasing temperature;

(v) both the 1 st and the 2nd peak blue shift with ageing and with increasing porosity;

(vi) intensity of the high energy peak increases where as the low energy peak intensity decreases with increasing temperature;

(vii) the intensity and peak position of the very low intensity 3rd peak appears to be unaffected by temperature, porosity, and ageing; and

(viii) for the low porosity samples $(P<80 \%)$, multiple peaks are not observed but the single peak intensity decreases and exhibits a red spectral shift [ $\sim 0.16 \mathrm{eV}$ for the sample shown in Fig. 4(d)] with increase in temperature form $10-300 \mathrm{~K}$.

The above features for the 1 st and the 2 nd peaks of the high porosity samples are clearly evident from Figs. 5(a)-5(c).

We first discuss the transition mechanism of the high porosity samples and consider two different possibilities. First, it is tempting to assign the 1st (high energy) peak centered around $2.7 \mathrm{eV}$ to originate from nearly stoichiometric $\mathrm{SiO}_{2}$ as reported previously by several groups. ${ }^{33-36}$ The $2.7 \mathrm{eV} \mathrm{PL}$ peak in nc-Si/ $\mathrm{SiO}_{2}$ system is well studied and is usually attributed to oxygen vacancies which induces very strong and anisotropic lattice distortion. ${ }^{36-38}$ However, the excitation energy required to obtain visible luminescence from $\mathrm{SiO}_{2}$ is usually much greater than $325 \mathrm{~nm}$, which is the exci-
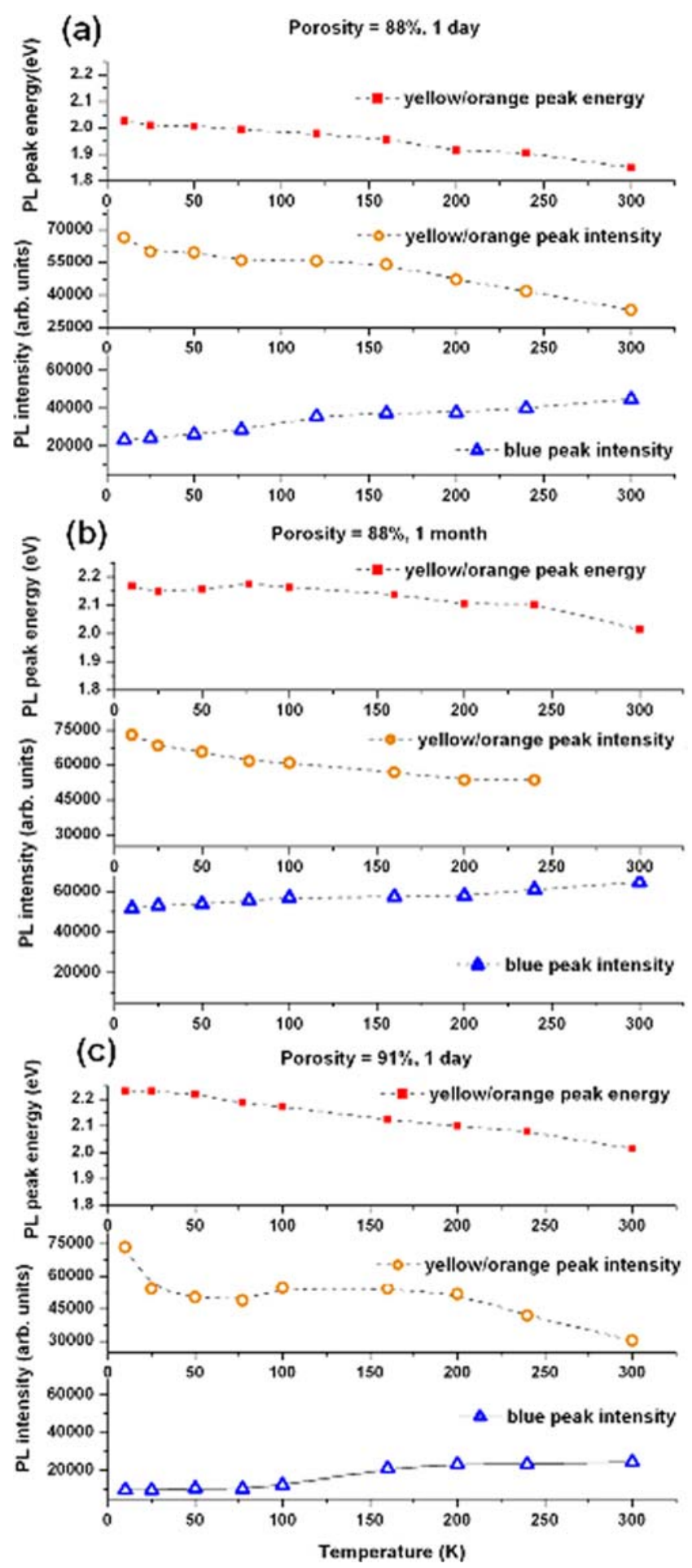

FIG. 5. (Color online) Variation of PL peak position and intensity with temperature for (a) the porous $\mathrm{Si}$ sample having porosity $\sim 88 \%$ prepared with $J=80 \mathrm{~mA} / \mathrm{cm}^{2}, t=15 \mathrm{~min}$, and $[\mathrm{HF}]=24 \%$ and dried in clean atmosphere for 1 day; (b) for the same sample after ageing for 1 month; and (c) for the sample with porosity $91 \%$ prepared with $J=100 \mathrm{~mA} / \mathrm{cm}^{2}, t=15 \mathrm{~min}$, $[\mathrm{HF}]=24 \%$ and aged for 1 day.

tation energy in our case, and is not enough to create nonequilibrium carriers across the bandgap of $\mathrm{SiO}_{2} \cdot{ }^{39} \mathrm{But}$, in the presence of non-stoichiometric suboxides of $\mathrm{Si}$, the bandgap is much smaller and may produce non-equilibrium carriers under UV excitation. ${ }^{40}$ Therefore, the possibility of such 
transitions cannot be ruled out. Furthermore, the absence of the high energy peak in the low porosity sample [Fig. 4(d)] extends support to this possibility as we see from the TEM and the AFM images that the oxide layers surrounding the nc-Si is visibly thick for high porosity samples [in fact, the nc-Si appears to be embedded in an oxide matrix in Fig. 1(c)] thereby increasing the possibility of existence of suboxides of Si in such samples.

According to this proposition, the 2nd (yellow/orange) peak (of the high porosity samples) may be assigned to band-to-band transitions and the red shift of the PL peak may be explained by temperature dependent change in the bandgap of $\mathrm{nc}-\mathrm{Si}^{41}$ The mechanism of the temperature induced bandgap shrinkage is well reported and explained in terms of change of lattice parameters and electron-phonon interaction. ${ }^{42}$ Quantitatively, the variation of the band-edge energy with temperature for semiconducting materials is most often accounted by the semi empirical Varshni equation (which is valid for bulk), ${ }^{43}$ or with other similar empirical relations suggested by other authors. ${ }^{44,45}$

In the framework of this possibility, the decrease in PL intensity of this 2 nd peak with increasing temperature may be qualitatively accounted due to increase of the probability of the non-radiative recombination channels. In addition, the observed increase in the $2.7 \mathrm{eV}$ band of the PL intensity with temperature seems to favor defect induced transitions as being responsible for this peak. The variation of the integrated PL intensity and the full width at half maxima (FWHM) of the PL spectra with temperature for the $88 \%$ porous sample, aged for 1 day are shown in Fig. 6. This variation could be apparently related to the enhanced surface diffusion of carriers towards radiative recombination centers which is supported by two previously suggested models; a non-bridging oxygen hole centre $\left(\mathrm{NBOH} ; \equiv \mathrm{Si}-\mathrm{O}^{\bullet}\right)^{46}$ and the triplet to ground state transition of a neutral oxygen vacancy $(\mathrm{NOV} ; \equiv \mathrm{Si}-\mathrm{Si} \equiv)$. $^{39,47}$

The integrated PL intensity $\left(I_{\text {int }}\right)$ of the localized exciton transition can be well described by the thermal activation process, defined by the equation: ${ }^{48} \ln \left(I_{\text {int }}\right)=\ln \left(I_{0}\right)-\left(\frac{E_{a c t}}{k}\right) \frac{1}{T}$,

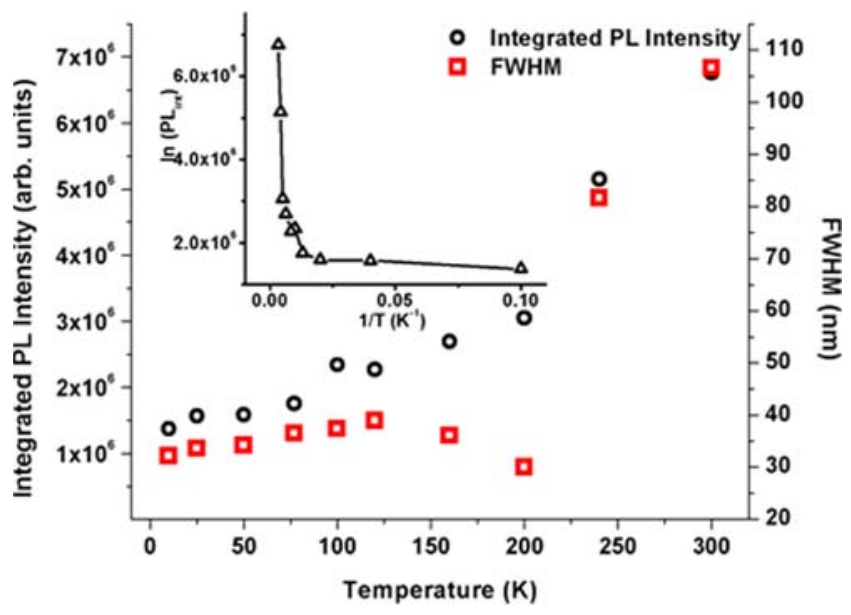

FIG. 6. (Color online) Variation of the integrated PL intensity and FWHM with temperature of the blue peak for the sample with porosity $88 \%$ and aged for 1 day. The plot of $\ln \left(I_{\text {int }}\right)$ vs. $1 / \mathrm{T}$ is shown in the inset. where, $E_{\text {act }}$ is the activation energy and $k$ is the Boltzmann constant. However, the inset shown in Fig. 6 clearly reveals that the variation of $\ln \left(I_{\text {int }}\right)$ with $1 / T$ is non-linear thereby suggesting that the features of the PL band at $\sim 2.7 \mathrm{eV}$ cannot be completely explained in terms $\mathrm{NOV}$ and/or $\mathrm{NBOH}$, in which case the electrons need to migrate to form selftrapped-excitons with thermally activated hopping.

In addition to the above, the first possibility also fails to satisfactorily explain the following observations: (1) the relatively large red spectral shift $(\sim 170$ and $220 \mathrm{meV}$ for 1-day aged samples with porosities $88 \%$ and $91 \%$, respectively) of the 2nd peak cannot be accounted for only by temperature dependent bandgap variation which is reported to be of the order of $50 \mathrm{meV}$ for bulk and $\sim 60 \mathrm{meV}$ for nc-Si for similar variation of temperature $^{41}$ and (2) the blue shift of the higher energy edge of the 1 st peak $(2.68-2.74 \mathrm{eV})$ with ageing and with increase in porosity.

Therefore, we consider a second possibility. In this case, for the oxidized samples (as all the samples were water rinsed and aged in ambient atmosphere), there are two competitive transition processes. Under UV excitation, electronhole (e-h) pairs are created in the nc-Si core. First, the photoexcited e-h pairs relax to the conduction band and valence band edges, respectively, and then recombine radiatively emitting higher energy (blue) photon. This accounts for the origin of the 1st peak. Second, the photo excited electron relaxes to the conduction band edge and the hole relaxes to the interface states present near the valence band edge. In other words, the photo-excited holes are captured by the interface states and subsequent radiative recombination emits photon with lower energy (than the band-to-band transition). This process is responsible for the 2 nd peak. However, just this proposition itself does not explain the characteristics of the temperature dependent PL spectra. In an attempt to do so, the effect of temperature on the first two processes has to be considered.

Focusing on the 2nd mechanism, there is a possibility that the captured hole will recombine with thermally excited electron from the valence band edge (and hence creating a hole in the valence band). Therefore, with increasing temperature, the probability of the 2 nd transition process decreases, while on the other hand the probability of band-to-band transition increases leading to simultaneous decrease in intensity of the low energy peak and increase in the high energy peak.

This proposition also explains the red spectral shift of the 2nd peak with increase in temperature. As temperature increases, more and more electrons will be thermally excited from the valence band to the interface states shown and marked by the shaded region depicting interface states filled with electrons in Fig. 7. Hence, with increasing temperature, the width of the shaded region is expected to increase leading to red spectral shift of the yellow orange peak. This phenomenon obviously couples with the temperature related bandgap modification which in association affects a shift of the order of $170-220 \mathrm{meV}$ for temperature varying from 10 to $300 \mathrm{~K}$. Therefore, for these samples, the variation of PL (or more precisely with the PL excitation) peak energies with temperature is not expected to fit either with the Varshni equation ${ }^{43}$ or with the other empirical relations. ${ }^{45,46}$ 


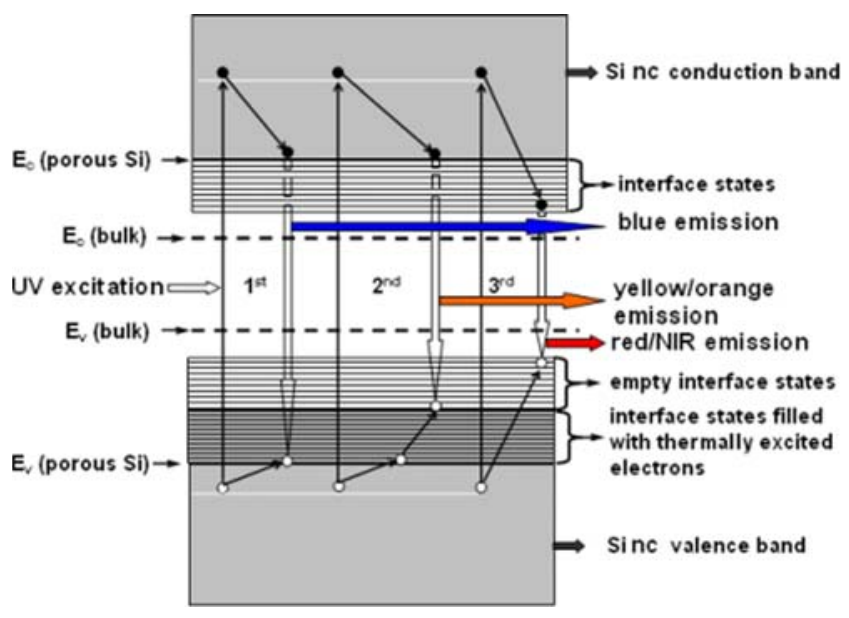

FIG. 7. (Color online) Schematic band diagram showing the different transition paths accounting for the different PL peaks in the temperature dependent spectra of porous $\mathrm{Si}$.

However, this mechanism cannot successfully explain the apparent temperature invariance of the high energy edge of the 1st PL peak. The non-Gaussian nature of the peak suggests that it is rather unlikely that the origin of this peak is purely due to band-to-band transition. If we assume that band-to-band transition is the sole contributor for this peak, then we would expect a temperature dependent red spectral shift due to bandgap shrinkage. ${ }^{43,45,46}$ Although it has been previously noted that this peak tends to broaden and form a plateau with increase in temperature, we see on closer inspection of the peak, as shown in Fig. 8 that the peak energy is nearly constant up to $120 \mathrm{~K}$ and after that it does exhibit a small $(\sim 40 \mathrm{meV})$ red shift. However, due to the formation of the plateau with a rather arbitrary shape, it is neither possible to uniquely identify the peak energy nor estimate the extent of the shift.

These observations prompt us to believe that the 1 st peak is due to the convoluted contribution of band-to-band and defect related transitions. This explains why we do not

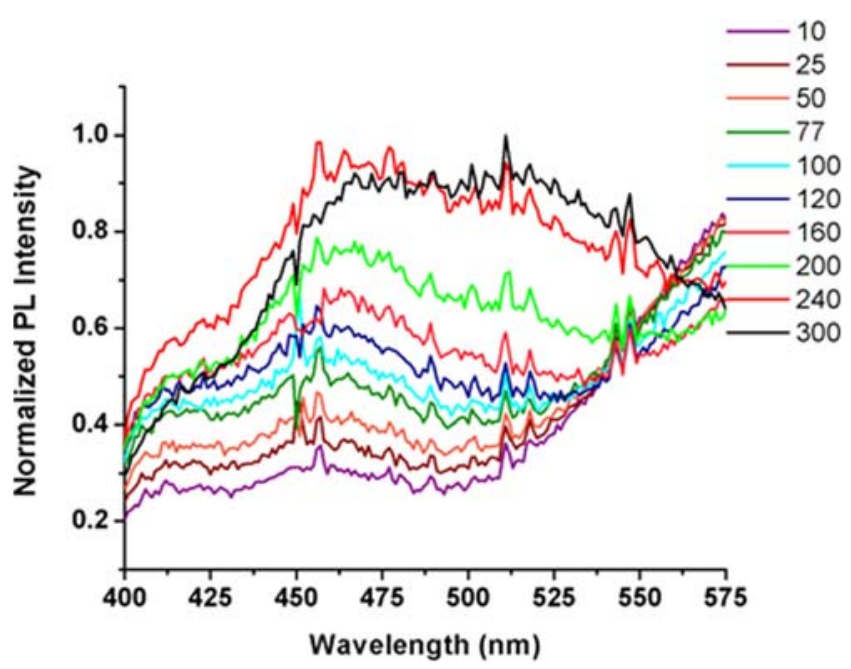

FIG. 8. (Color online) Normalized PL spectra (temperature dependent) showing only the high energy peak for the sample with porosity $88 \%$ and aged for 1 day. see either a purely thermally activated hopping like transition (necessary to justify NOV or NBOH models) or Gaussian peaks that exhibit a red spectral shift (in keeping with band transitions). In addition, this explains the blue shift of the 1st peak with increase in porosity. The band edges of nc-Si are affected by changes in porosity due to changes in the particle size of the ncs. Thus, for the higher porosity samples prepared with high current densities, the peak positions of both the 1st and 2nd peaks exhibit blue shift. This is because of the quantum confinement contribution which opens up the bandgap with reduction in particle size. Moreover, ageing of a given sample also widens up the bandgap thereby affecting a blue shift of both the peaks at any given temperature.

There is another possible path of radiative recombination indicated in the band structure (shown in Fig. 7) described above to explain the red/NIR peak. A hole can be relaxed at the interface states above the valence band (as in case of 2nd process), as well as the electron can be captured (or relaxed) at the interface states near the conduction band edge. This may lead to even lower energy PL emission in the red/NIR emission $(\sim 800 \mathrm{~nm})$. Definitely, the probability of this process is lesser than that of the 2 nd process since the mobility of hole is less than that of electrons. So, the capture cross section for holes is higher than that of electrons for equal density of electron and hole capturing states. Hence, the relaxation of electrons by the interface states is less probable than that of holes leading to very low intensity of the peak $\sim 800 \mathrm{~nm}$. However, if by some means, the mobilities of the two types of carriers can be made comparable to each other then the intensity of this peak may increase. This may be achieved either by reducing the dimension of the nc, so that the mobility of the electron is almost frozen, or by modifying the interface in such a manner that the density of electron capturing states becomes much higher than that of hole capturing states. The relative increase of intensity of this peak (compared to the 1st and the 2nd peaks) for aged sample and for the one with higher porosity supports the proposition. Both with ageing and with increase in porosity there is a further reduction in size which effectively increases the electron capture cross section. The constant position of this peak for 2 different porosities may be explained by the fact that the position of the interface states inside the bandgap is almost independent of particle size. ${ }^{10,49}$ The transition mechanism proposed is summarized by the different paths described in Fig. 7.

The argument proposed to explain the transition mechanisms in high porosity samples also explains the characteristics of the low porosity porous Si. Since lower porosity corresponds to larger crystallite sizes and much less oxide coverage (as evidenced from TEM images) contribution due to transitions from oxides and/or suboxides of $\mathrm{Si}$ and quantum confined states are much less compared to the oxide related interface states. Consequently, the PL spectra are dominated by transitions mediated by the interface states. The small red spectral shift and the continuous decrease in intensity with increase in temperature re-affirm the proposed mechanism. The relatively large crystallite sizes also accounts for higher relative mobility of the electrons compared to the holes and hence the 3rd peak observed in the 
highly porous samples are understandably absent in the PL characteristics of the lower porosity samples.

Thus, the present study, on one hand, enables us to experimentally identify and distinguish between the different transition mechanisms responsible for radiative emission from porous $\mathrm{Si}$ nanostructures and on the other hand, resolves the long-standing contradictory reports on the temperature dependent PL characteristics of porous Si.

\section{CONCLUSIONS}

In summary, temperature dependent PL characteristics of porous Si nanostructures reveal that two competitive processes involving band-to-band and oxide related interface mediated transitions are responsible for radiative emission from porous Si. Structural investigations reveal that the porous $\mathrm{Si}$ nanostructures consist of nanorods and nanodots of randomly oriented nc-Si surrounded by oxide shell. The higher porosity $(P>80 \%)$ samples formed with larger current densities have much smaller average nc-Si core $(\sim 1-6 \mathrm{~nm})$ and their PL spectra are characterized by the appearance of multiple peaks which become distinctly distinguishable at lower temperatures. The lower porosity samples evidently consist of comparatively larger nc-Si and have single peaks in their emission spectra even at low temperatures $(10 \mathrm{~K})$. A systematic investigation of the variation of intensity and PL peak position with temperature (10-300 K) of the differently prepared and aged samples allows us to experimentally distinguish the contribution of quantum confinement induced widened bandgap related transitions and oxide, interfacial defect states mediated radiative recombination. The findings also resolves the long-standing conflicting reports on the temperature dependent PL characteristics of nanostructured porous $\mathrm{Si}$.

\section{ACKNOWLEDGMENTS}

This work has been partly supported by Department of Science and Technology (DST), India (Ref no. DST/INT/ AUS/PROJ/T-2/08) and Centre for Research Excellence in Fabrionics, Indo-US Science and Technology Forum.

${ }^{1}$ L. T. Canham, Appl. Phys. Lett. 57, 1046 (1990).

${ }^{2}$ Structural and Optical Properties of Porous Silicon Nanostructures, edited by G. Amato, C. Delerue, and H.-J, von Bardeleben (Gordon and Breach science publishers, Amsterdam, 1997).

${ }^{3}$ Properties of Porous Silicon, edited by L. T. Canham (INSPEC, The Institution of Electrical Engineers, London, 1997).

${ }^{4}$ Porous Silicon, Z. C. Feng and R. Tsu (World Scientific, Singapore, 1994).

${ }^{5}$ A. G. Cullis, L. T. Canham, and P. D. J. Calcott, J. Appl. Phys. 82, 909 (1997).

${ }^{6}$ O. Bisi, S. Ossicini, and L. Pavesi, Surf. Sci. Rep. 38, 1 (2000).

${ }^{7}$ H. Föll, M. Christophersen, J. Carstensen, and G. Hasse, Mater. Sci. Engg. R 39, 93 (2002).

${ }^{8}$ C. Delerue, G. Allan, and M. Lannoo, Phys. Rev. B 48, 11024 (1993).

${ }^{9}$ S. Ogüt, J. R. Chelikowsky, and S. G. Louie, Phys. Rev. Lett. 79, 1770 (1997).

${ }^{10}$ M. V. Wolkin, J. Jorne, P. M. Fauchet, G. Allan, and C. Delerue, Phys. Rev. Lett. 82, 197 (1999).

${ }^{11}$ P. McCord, S.-L. Yau, and A. J. Bard, Science 257, 68 (1992).
${ }^{12}$ M. M. Das, M. Ray, N. R. Bandyopadhyay, and S. M. Hossain, Mater. Chem. Phys. 119, 524 (2010).

${ }^{13}$ L. Bagolini, A. Mattoni, G. Fugallo, and L. Colombo, Phys. Rev. Lett. 104, 176803 (2010).

${ }^{14}$ S. Godefroo, M. Hayne, M. Jivanescu, A. Stesmans, M. Zacharias, O. I. Lebedev, G. Van tendeloo, and V. V. Moshchalkov, Nature Nanotechnol. 3, 174 (2008).

${ }^{15}$ R. Soulairol and F. Cleri, Solid State Sci. 12, 163 (2010).

${ }^{16}$ F. Rongchuan, L. Qingshan, and C. Jingbiao, Chin. Phys. Lett. 9, 438 (1992).

${ }^{17}$ N. Ookubo and S. Sawada, Phys. Rev. B 51, 17526 (1995).

${ }^{18}$ S. Finkbeiner and J. Weber, Thin Solid Films 255, 254 (1995).

${ }^{19}$ S. Gardelis, J. S. Rimmer, P. Dawson, B. Hamilton, R. A. Kubiak, T. E. Whall, and E. H. C. Parker, Appl. Phys. Lett. 59, 2118 (1991).

${ }^{20}$ X. L. Zeng, W. Wang, and H. C. Chen, Appl. Phys. Lett. 60, 986 (1992).

${ }^{21}$ Z. Y. Xu, M. Gral, and M. Gross, Appl. Phys. Lett. 60, 1375 (1992).

${ }^{22}$ Y. K. Xu and S. Adachia, J. Appl. Phys. 107, 123520 (2010).

${ }^{23}$ S. M. Hossain, J. Das, S. Chakraborty, S. K. Dutta, and H. Saha, Semicond. Sci. Technol. 17, 55 (2002).

${ }^{24}$ J. Rouquerol, D. Avnir, C. W. Fairbridge, D. H. Everett, J. H. Haynes, N. Pernicore, J. D. F. Ramsey, K. S. W. Sing, and K. K. Unger, Pure Appl. Chem. 66, 1739 (1994).

${ }^{25}$ M. Ray, S. Ganguly, M. Das, S. Datta, N. R. Bandyopadhyay, and S. M. Hossain, Mater. Manuf. Processes 24, 83 (2009).

${ }^{26}$ M. Ray, T. S. Basu, A. Jana, N. R. Bandyopadhyay, S. M. Hossain, A. K. Pramanick, and R. F. Klie, J. Appl. Phys. 107, 064311 (2010).

${ }^{27}$ M. Ray, S. Sarkar, N. R. Bandyopadhyay, S. M. Hossain, and A. K. Pramanick, J. App. Phys. 105, 074301 (2009).

${ }^{28}$ M. A. Salem, H. Mizuta, and S. Oda, Appl. Phys. Lett. 85, 3262 (2004).

${ }^{29}$ C. H. Lei, K. Ouzineb, O. Dupont, and J. L. Keddie, J. Colloid Interf. Sci. 307, 56 (2007).

${ }^{30}$ L. T. Canham, "Progress Towards Understanding and Exploiting the Luminescent Properties of Highly Porous Silicon," in Optical Properties of Low Dimensional Silicon Structures, NATO ASI Series, edited by D. C. Benshael, L. T. Canham, and S. Ossicini (Kluwer Academic Publishers, Dordrecht, 1993), Vol. 244, pp. 81-94.

${ }^{31}$ L. T. Canham, M. R. Houlton, W. Y. Leong, C. Pickering, and J. M. Keen, J. Appl. Phys. 70, 422 (1991).

${ }^{32}$ D. W. Cooke, R. E. Muenchausen, B. L. Bennett, L. G. Jacobsohn, and M. Nastasi, J. Appl. Phys. 96, 197 (2004).

${ }^{33}$ R. Tohmon, Y. Shimogaichi, H. Mizuno, Y. Ohki, K. Nagasawa, and Y. Hama, Phys. Rev. Lett. 62, 1388 (1989).

${ }^{34}$ L. Skuja, J. Non-Cryst. Solids 149, 77 (1992)

${ }^{35}$ S. Sato, H. Ono, S. Nozaki, and H. Morisaki, Nanostruct. Mater. 5, 589 (1995).

${ }^{36}$ L. S. Liao, X. M. Bao, X. Q. Zheng, N. S. Li, and N. B. Min, Appl. Phys. Lett. 68, 850 (1996).

${ }^{37}$ M. Bertino, A. Corazza, M. Martini, A. Mervic, and G. Spinolo, J. Phys. Condens. Matter 6, 6345 (1994).

${ }^{38}$ L. S. Liao, X. M. Bao, N. S. Li, X. Q. Zheng, and N. B. Min, J. Lumin. 68, 199 (1996)

${ }^{39}$ L. Tsybeskoy, Ju. V. Vandyshev, and P. M. Fauchet, Phys. Rev. B 49, 7821 (1994).

${ }^{40}$ H. R. Philipp, J. Phys. Chem. Solids 32, 1935 (1971).

${ }^{41}$ M. L. Brongersma, P. G. Kik, A. Polman, K. S. Min, and H. A. Atwater, Appl. Phys. Lett. 76, 351 (2000).

${ }^{42}$ A. S. Zubrilov, S. A. Nikishin, G. D. Kipshidze, V. V. Kuryatkov, H. Temkin, T. I. Prokofyeva, and M. Holtz, J. Appl. Phys. 91, 1209 (2002).

${ }^{43}$ Y. P. Varshni, Physica 34, 149 (1967).

${ }^{44}$ K. P. O’Donnell and X. Chen, Appl. Phys. Lett. 58, 2924 (1991).

${ }^{45}$ M. Cardona, T. A. Meyer, and M. L. W. Thewalt, Phys. Rev. Lett. 92, 196403 (2004).

${ }^{46}$ A. J. Kenyon, P. F. Trwoga, C. W. Pitt, and G. Rehm, J. Appl. Phys. 79, 9291 (1996).

${ }^{47}$ G. Lucovsky, J. Yang, S. S. Chao, J. E. Tyler, and W. Czubatyj, Phys. Rev. B 28, 3225 (1983).

${ }^{48}$ N. Nepal, J. Li, M. L. Nakarmi, J. Y. Lin, and H. X. Jiang, Appl. Phys. Lett. 88, 062103 (2006).

${ }^{49}$ T. Makimura, Y. Kunii, N. Ono, and K. Murakami, Appl. Surf. Sci. 127129, 388 (1998). 